Research Paper

\title{
Datumetine Preferentially Upregulates N-methyl-D- aspartate Receptor Signalling Pathways in Different Brain Regions of Mice
}

Azeez Olakunle Ishola ${ }^{1^{*}}$ (D), Adedeji Enitan Adetunji ${ }^{1}$ (D), Isaac Chukwunwike Abanum ${ }^{1}$, Adesola Akorede Adeyemi ${ }^{1}$, Caleb Kenechukwu Faleye $^{1}$, Jane Babale Martins', Nnenna Chimdalu Ogbe ${ }^{1}$, Toluwalase Cherish Ogundipe ${ }^{1}$, Karen Ezichi Okewulonu ${ }^{1}$, Umo Emmanuel Okon $^{1}$, Deborah Irenoise Ovbude ${ }^{1}$, Richard Yomi Akele ${ }^{2}$ (i), Naeemat Tolulope Omotade ${ }^{3}$, Moyosore Salihu Ajao ${ }^{4}$ (i)

1. Department of Anatomy, College of Medicine and Health Sciences, Afe Babalola University Ado-Ekiti, Nigeria.

2. Department of Medical Laboratory Science, College of Medicine and Health Sciences, Afe Babalola University, Ado-Ekiti, Nigeria.

3. Department of Anatomy, College of Health Technology, Ijero-Ekiti, Nigeria.

4. Department of Anatomy, University of Ilorin, Ilorin, Nigeria.

\begin{tabular}{|c|c|}
\hline $\begin{array}{l}\text { Use your device to scan } \\
\text { and read the article online }\end{array}$ & ditation Ishola, A. O., Adetunji, A, E., Abanum, I. C., Adeyemi, A. A., Faleye, C. K., \& Martins, J.B., et al. (2023). \\
\hline 口ifirita & $\begin{array}{l}\text { Datumetine Preferentially Upregulates N-methyl-D-aspartate Receptor Signalling Pathways in Different Brain Re- } \\
\text { gions of Mice. Basic and Clinical Neuroscience, 14(1), 103-116. http://dx.doi.org/10.32598/bcn.2021.3397.1 }\end{array}$ \\
\hline actistis & d.i'http://dx.doi.org/10.32598/bcn.2021.3397.1 \\
\hline
\end{tabular}

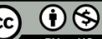

Article info:

Received: 13 May 2021

First Revision: 15 Jun 2021

Accepted: 30 Jun 2021

Available Online: 01 Jan 2023

Keywords:

Datumetine, N-methyl-Daspartate receptor (NMDAR), Dizocilpine (MK-801), Hippocampus, Cerebellum, Prefrontal cortex (PFC)

\section{A B S T RA C T}

Introduction: We previously reported that datumetine possesses binding affinity with $\mathrm{N}$-methylD-aspartate receptor (NMDAR) and that 14-day exposure to datumetine altered NMDAR signaling by mimicking glutamate toxicity. Here, we investigated the potential neuroprotective effect of a single shot of a low dose of datumetine administration in $\mathrm{BALB} / \mathrm{c}$ mice.

Methods: 30 male adult BALB/c mice were used for the study. The mice were randomly divided into three groups of ten mice each with an intraperitoneal injection of $0.1 \mathrm{~mL}$ of $10 \%$ DMSO for the Vehicle group, Datumetine group were administered $0.1 \mathrm{mg} / \mathrm{kg}$ body weight (bw) of datumetine and MK-801+Datumetine group were administered $0.5 \mathrm{mg} / \mathrm{kg}$ bw of MK801 (to block NMDAR) followed by $0.1 \mathrm{mg} / \mathrm{kg}$ bw of datumetine after 30 minutes. 24 hours after administration, mice were euthanized in an isoflurane chamber followed by perfusion with $1 \mathrm{X}$ PBS. Brains were excised and stored at $-20^{\circ} \mathrm{C}$ till further processing. Mice designated for IHC were further perfused with 4\% PFA and brain excised and stored in 4\% PFA till further processing. NMDAR signalling molecules expression was evaluated in frozen brain samples and the fixed brain samples were stained for neuron, vGlut and NMDAR subtypes. Results: Relative to vehicle (Veh), datumetine downregulate calcium calmodulin kinase II alpha (CamKII $\alpha$ ) expression in the hippocampus and prefrontal cortex (PFC) but not in the cerebellum, cyclic AMP response element binding protein (CREB) was also upregulated only in the PFC but phosphorylated CREB (pCREB) was also upregulated in three brain regions observed, while brain-derived neurotrophic factor (BDNF) was only upregulated in hippocampus and PFC of Datumetine relative to vehicle (Veh). On the other hand, dizocilpine (MK-801) reversed some of the effects of datumetine in the observed brain regions. No major histological alterations were observed in the different brain regions immunohistochemically.

Conclusion: We conclude that a low dose of datumetine moderately enhances NMDAR activity. This showed the neuroprotective potentials of low datumetine exposure.

\footnotetext{
* Corresponding Author:

Azeez Olakunle Ishola, PhD.

Address: Department of Anatomy, College of Medicine and Health Sciences, Afe Babalola University, Ado-Ekiti, Nigeria.

Tel: +23 (47) 064267649

E-mail:ao.ishola@abuad.edu.ng
} 


\section{Highlights}

- Datumetine activates n-methyl-D-aspartate receptor (NMDAR) activity in different brain regions

- Datumetine increases the activity of glutamate transmission

- Datumetine alters the level of major NMDAR subtypes in the brain

\section{Plain Language Summary}

Datumetine is a compound obtained from the Datura metel which is a psychoactive plant. Its users suffer from amnesia during intoxication. We have shown from our previous study that datumetine can bind with NMDAR and alter its activity. Here we demonstrated that giving a little amount of datumetine can modulate the activity of NMDAR in a way to be beneficial to the brain.

\section{Introduction}

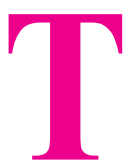

he World Health Organization (WHO) reported that central nervous system $(\mathrm{CNS})$ disorders account for more than $6 \%$ of disabilities, with lower-middle-income countries suffering more burden (Moritz et al., 1997).

Africa is expected to experience more mortality from these CNS disorders and is projected to increase in 2030 (Moritz et al., 1997). The brain is a homeostatic working machine, perturbations in the brain neurotransmitters affect its overall working system (Ishola et al., 2020).

$\mathrm{N}$-methyl-D-aspartate receptor (NMDAR) is an ionotropic glutamate receptor expressed ubiquitously in the brain (Kennedy, 2010; Sapkota et al., 2019; Sattler et al., 2000). It belongs to the ionotropic class of glutamate receptors (Montes de Oca Balderas, 2018; Reiner \& Levitz, 2018). NMDAR activation by glutamate leads to an influx of calcium ions $\left(\mathrm{Ca}^{2+}\right)$ that activates a diverse range of intracellular signaling cascades (Cho et al., 2012; Kennedy, 2010; Montes de Oca Balderas, 2018; Sapkota et al., 2019). This is why NMDAR is regarded as an electrochemical coupling (Zhu et al., 2016). Disruptions in the NMDAR activity in the brain are associated with diverse neuropsychiatric disorders (Bartlett \& Wang, 2013; Das et al., 2018; Henson et al., 2017; Ni et al., 2018; Vishnoi et al., 2015).

Plant compounds have played a significant role in drug discovery over the years. The use of plant compounds for medicinal purposes is common in Africa and dates back to prehistoric times. Previous work from our laboratory showed the potential of a new alkaloid from Datura metel (datumetine) potentiating NMDAR. Our previous study showed that datumetine possessed a binding affinity for NMDAR and prolong exposure in mice altered the NMDAR signaling pathway and hippocampal neurotransmitters system mimicking glutamate toxicity (Ishola et al., 2020). We propose that datumetine replaces glutamate at its binding site on NMDAR and has a similar role to glutamate binding.

This study aims to investigate whether a single dose of datumetine could mimic synapto-nuclear signaling in the brain. This is because low exogenous glutamate exposure has been reported to be a model to enhance synaptic plasticity (Bartlett \& Wang, 2013; Kennedy, 2010; Lu \& Malenka, 2012; Zhu et al., 2015) there have been few attempts to correlate these important areas of research. Here we review current knowledge of the various mechanisms of NMDAR-dependent synaptic plasticity that are shared with neuronal survival and death, while drawing comparisons with the proneurotrophin/ neurotrophin receptor and intracellular signaling systems. Our conclusion is that NMDAR-dependent LTP and long-term depression (LTD.

\section{Materials and Methods}

\section{Drug purchase and preparation}

Datumetine (CAS No.:67078-20-0) was a gift from Dr. Ogundele of Louisiana State University USA. Dizocilpine (MK-801) (CAS No.:77086-21-6) was procured from MedChemExpress (MCE) USA. The drugs were all dissolved in 10\% dimethylsulfoxide (DMSO) to make a $1 \mathrm{mg} / \mathrm{mL}$ stock solution. 


\section{Animal care}

Thirty male adult BALB/c mice were procured from the University Animal Care Center. The mice were housed in standard laboratory conditions of 12 hours alternating light and dark cycles. They were kept in standard cages of five mice per cage. The mice were allowed free access to feed and water.

The mice were randomly divided into three groups of ten mice. The vehicle (Veh) group was administered 0.1 $\mathrm{mL}$ of $10 \%$ dimethyl sulfoxide (DMSO), datumetine group was administered $0.1 \mathrm{mg} / \mathrm{kg}$ body weight (BW) of datumetine (Ishola et al., 2021) while MK-801+ datumetine group were administered $0.5 \mathrm{mg} / \mathrm{kg} \mathrm{BW}$ of MK801 (to block NMDAR) (Svalbe et al., 2019) followed by $0.1 \mathrm{mg} / \mathrm{kg} \mathrm{BW}$ of datumetine after 30 minutes. All administration was done intraperitoneally.

\section{Animal sacrifice}

Twenty-four hours after the administration, the mice were euthanized using isoflurane in a desiccator. After the mice became unconscious, they were perfused transcardially with $10 \mathrm{mM}$ phosphate-buffered solution (1X PBS pH 7.4) to expel the blood. Five mice designated for protein assay had their brain excised, prefrontal cortex (PFC), hippocampus, and cerebellum of the excised brains were dissected out and placed in tubes containing 10 times the volume of radioimmunoprecipitation assay (RIPA) lysis buffer (HY-K1001 MCE, USA) to the weight of specimen and stored at $-20^{\circ} \mathrm{C}$ until further processing. Five mice designated for immunohistochemistry study were further perfused with $4 \%$ paraformaldehyde (PFA) after PBS and the brain was excised and stored in a specimen bottle containing $4 \%$ paraformaldehyde (PFA) and stored at $4{ }^{\circ} \mathrm{C}$ until further processing.

\section{Protein assay}

Brain samples were homogenized using a cold manual homogenizer on ice. Homogenates were centrifuged (using Prolab laboratory centrifuge Model TD4Z USA) at $1000 \mathrm{rpm}$ for 20 minutes at $4^{\circ} \mathrm{C}$. The supernatant was collected in another tube for the protein assay. The level of calcium calmodulin kinase II (CamKII), cyclic AMP response element-binding protein (CREB), phosphorylated CREB (pCREB) and brain-derived neurotrophic factor (BDNF) were assessed in the supernatant using enzyme-linked immunosorbent assay (ELISA) technique at $420 \mathrm{~nm}$ using Matrotech ELISA plate analyzer model, read well TOUCH SA. Table 1 presents details of ELISA kits. All procedures were performed strictly as stated by the manufacturer.

\section{Immunohistochemistry (IHC)}

The brain samples for Immunohistochemistry (IHC) were processed for paraffin embedding techniques and were sectioned sagittally at $10 \mu \mathrm{m}$ and placed on charged slides. Slides were incubated in an oven at $50^{\circ} \mathrm{C}$ for 20 minutes after which the antigens were retrieved. The slides were then triple stained for NeuN (neurons), vGlut1 (glutamate terminals), and GluN2A or GluN2B (NMDAR subtypes) using TripleStain IHC kit (ab183295) from Abcam USA. Table 2 presents the details of the primary antibody used.

\section{Microscopy}

The slides were viewed under a light microscope with an in-built camera (Opto-Edu China). The same areas were studied in all the brain slides and captured with an $\mathrm{x} 40$ objective lens. Twenty sections from each mouse were used for quantitative analysis using ImageView software, version x64, 3.7.10270.2017.1112 China

\section{Statistical analysis}

Quantitative data were expressed as Mean \pm SE. Data were analyzed using one-way analysis of variance (ANOVA) with the Tukey post hoc test which was significant $\mathrm{P}=0.05$. This was done using GraphPad prism software, version 5.0.

\section{Results}

Datumetine reduces calcium calmodulin kinase ii alpha (CamKIIa) expression in the hippocampus and prefrontal cortex (PFC) but not cerebellum

CamKII $\alpha$ is a holoenzyme associated with NMDAR that is phosphorylated upon NMDAR activation. The CamKII $\alpha$ level was not significantly altered in the hippocampus and PFC of mice exposed to a single datumetine dose and mice pre-treated with MK-801 compared to the Veh mice (Figure $1 \mathrm{~A}$ and B). This observation is different from the cerebellum of mice exposed to a single datumetine dose which showed a significant increase $\left({ }^{* *} \mathrm{P}<0.01\right)$ compared to the mice treated with the vehicle solution and MK-801 pre-treated mice (see Figure $1 \mathrm{C}$ ).

Datumetine does not alter the level of cyclic AMP response element binding protein (CREB) in Different cortical regions of mice

NMDAR activation leads to an influx of $\mathrm{Ca}^{2+}$ into the neurons, which leads to the activation of CREB and subsequently to the activation of downstream signal- 
Table 1. List and manufacturer details of ELISA kits used

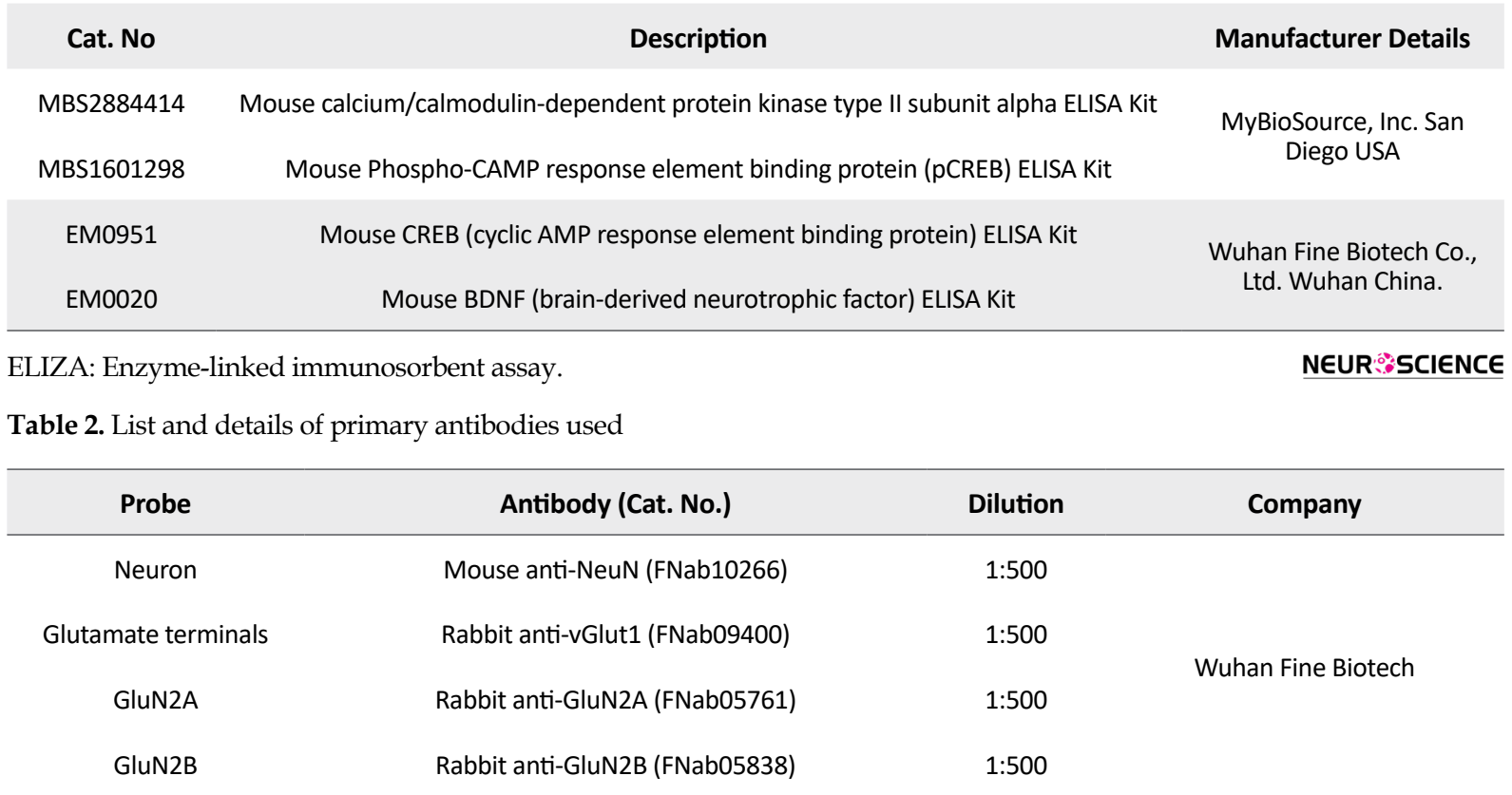

NEUR SCIENCE

ing molecules. No significant changes were observed in the CREB level of PFC and cerebellum of experimental mice (Figure $2 \mathrm{~B}$ and $2 \mathrm{C}$ ). While in the hippocampus, blocking of NMDAR with MK-801 before datumetine treatment significantly $\left({ }^{* * *} \mathrm{P}<0.001\right)$ increased the level of hippocampal CREB in the mice compared to Veh mice and datumetine mice (Figure $2 \mathrm{~A}$ ).
Datumetine favours cyclic AMP response element binding protein (CREB) Phosphorylation in different cortical regions of mice

Part of the mechanism of NMDAR signaling downstream is the phosphorylation of CREB at Serine 133. Exposure of mice to a single datumetine did not significantly increase pCREB level in the hippocampus and cerebellum compared to Veh mice (Figure $3 \mathrm{~A}$ and 3
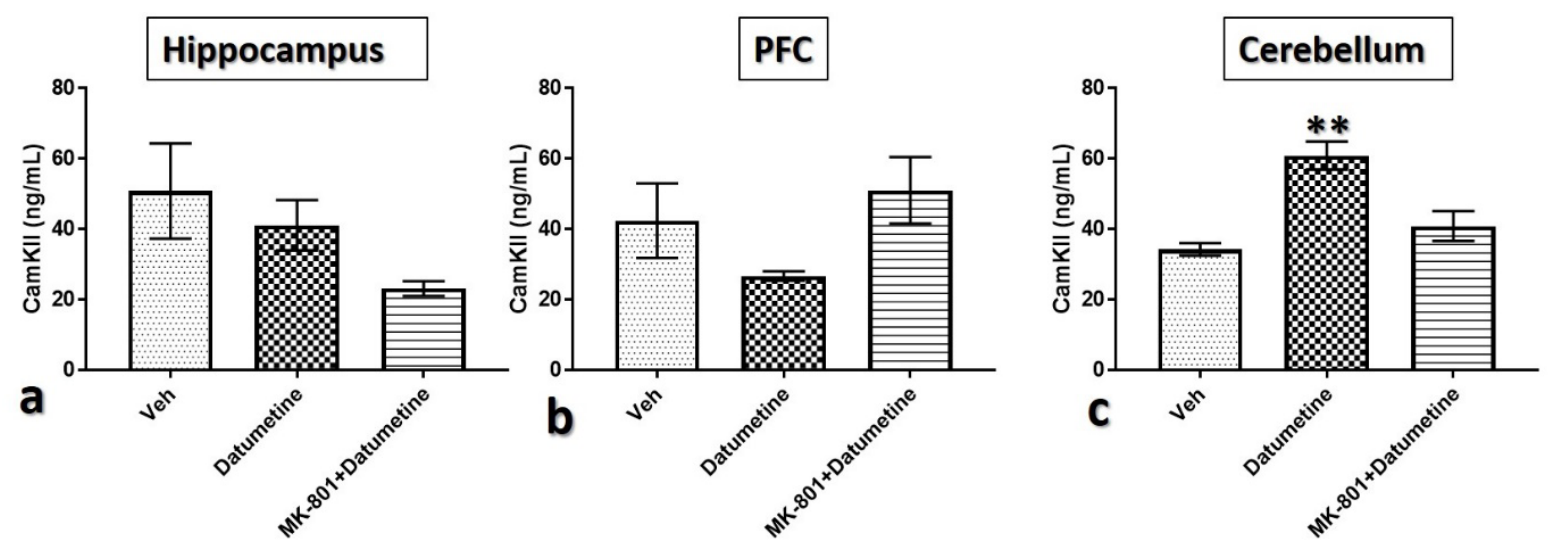

NEUR SCIENCE

Figure 1. Graphical representation of calcium calmodulin kinase II (CamKII) level in different brain regions

A) Hippocampal level of CamKII was reduced in datumetine and MK-801 pre-treated mice than Veh mice with no significant difference.

B) PFC level of CamKII was reduced in datumetine mice reversed in MK-801 pre-treated mice with no significant difference.

C) Cerebellar level of CamKII was significantly $\left({ }^{*} \mathrm{P}<0.01\right)$ increased in datumetine than Veh mice reversed by MK-801 pretreatment. 

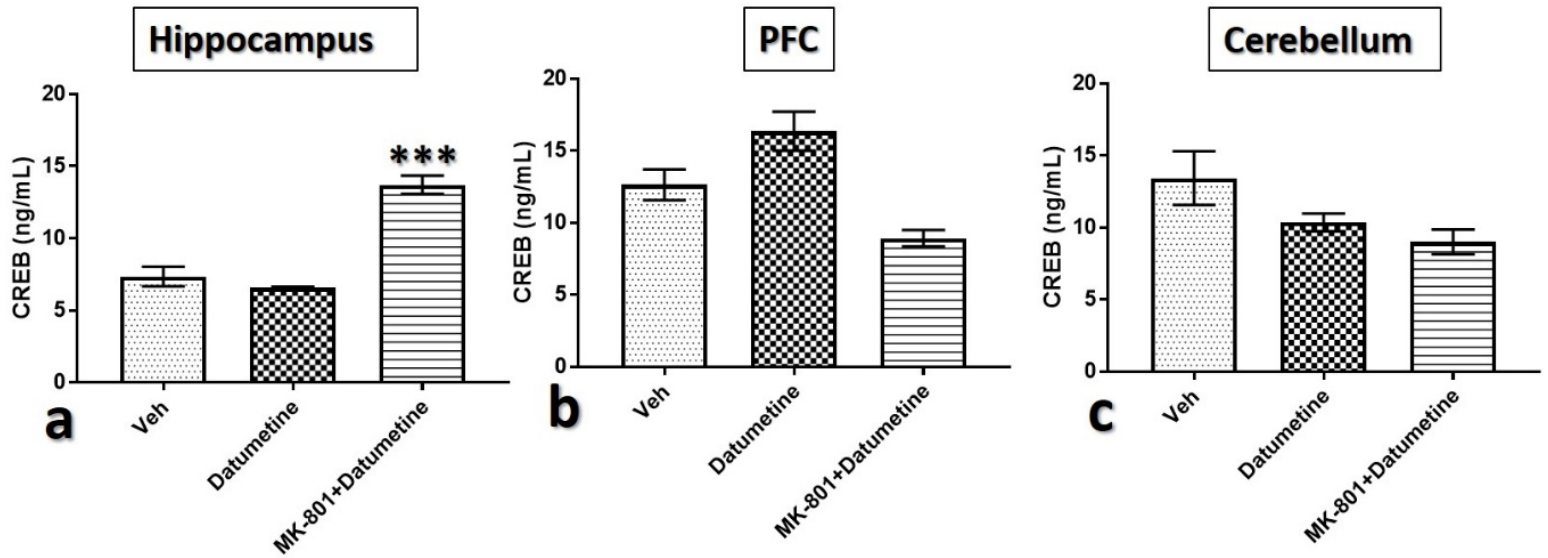

NEUR SCIENCE

Figure 2. Graphical representation of cyclic AMP response element binding protein (CREB) level in different brain regions

A) Hippocampal level of CREB was significantly $\left({ }^{* * *} \mathrm{P}<0.001\right)$ increased in $\mathrm{MK}-80+$ datumetine mice than Veh and datumetine mice.

B) PFC level of CREB was increased in Datumetine mice while MK-801+datumetine mice showed reduced CREB than Veh, though no significant difference was observed.

C) Cerebellar CREB level was reduced in all treated mice compared to Veh mice, though not significant.

C). In the PFC, the level of pCREB was significantly $(* * * \mathrm{P}<0.001)$ increased in datumetine mice compared to Veh mice (Figure $3 \mathrm{~B}$ ). MK-801 was able to reverse datumetine effect in the PFC as mice in MK-801+ datumetine group showed a significantly reduced level of pCREB compared to datumetine but not significantly compared to Veh mice (Figure 3 B).
Datumetine increased brain-derived neurotrophic factor (BDNF) level in the hippocampus and cerebellum but not prefrontal cortex (PFC)

In the synaptic-nuclear signaling pathway of NMDAR, pCREB translocates into the nucleus to initiate the expression of pro-survival genes, such as BDNF. The level of BDNF was significantly increased $(* * * \mathrm{P}<0.001)$ in the hippocampus and cerebellum of datumetine mice compared to Veh mice and MK-801 pre-treated mice
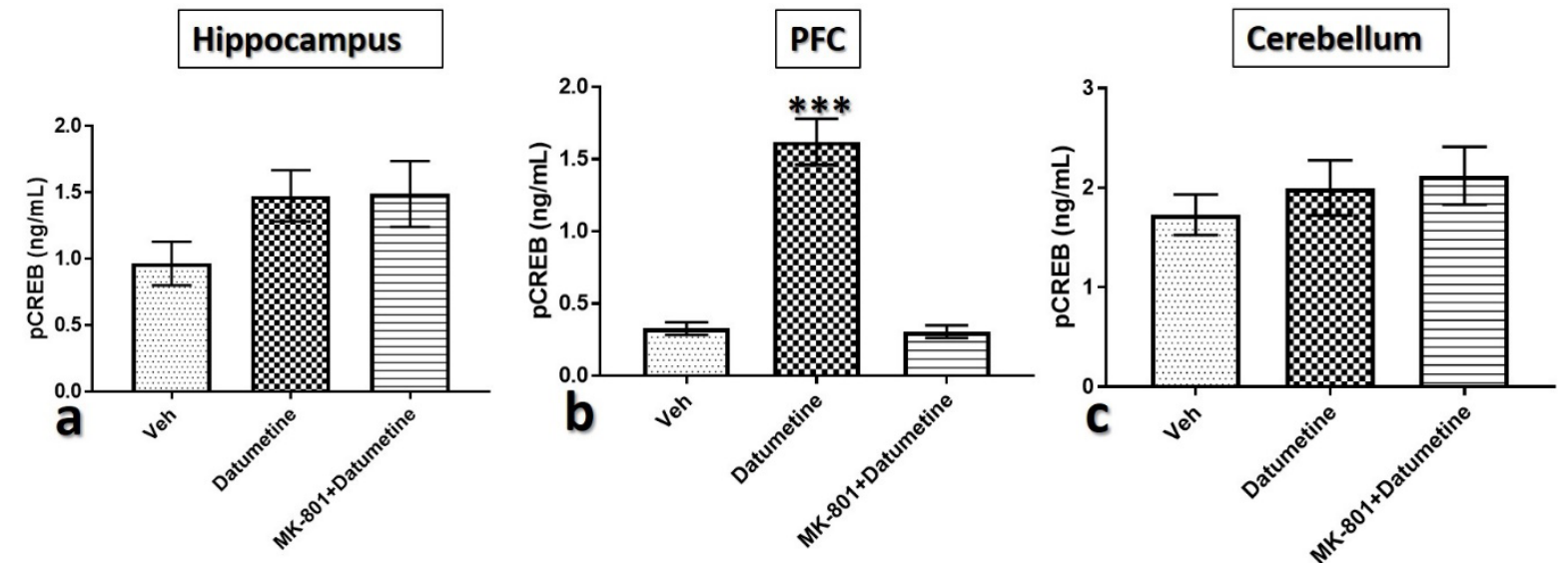

NEUR:SCIENCE

Figure 3. Graphical representation of cyclic AMP response element binding protein (pCREB) level in different brain regions

A) Hippocampal pCREB level was increased in all treated mice than Veh mice, though not significant.

B) PFC level of pCREB was significantly (***P<0.001) higher than Veh and MK-801+datumetine mice.

C) Cerebellar level of pCREB was increased in Datumetine and MK-801 pre-treated mice than Veh, though not significant. 

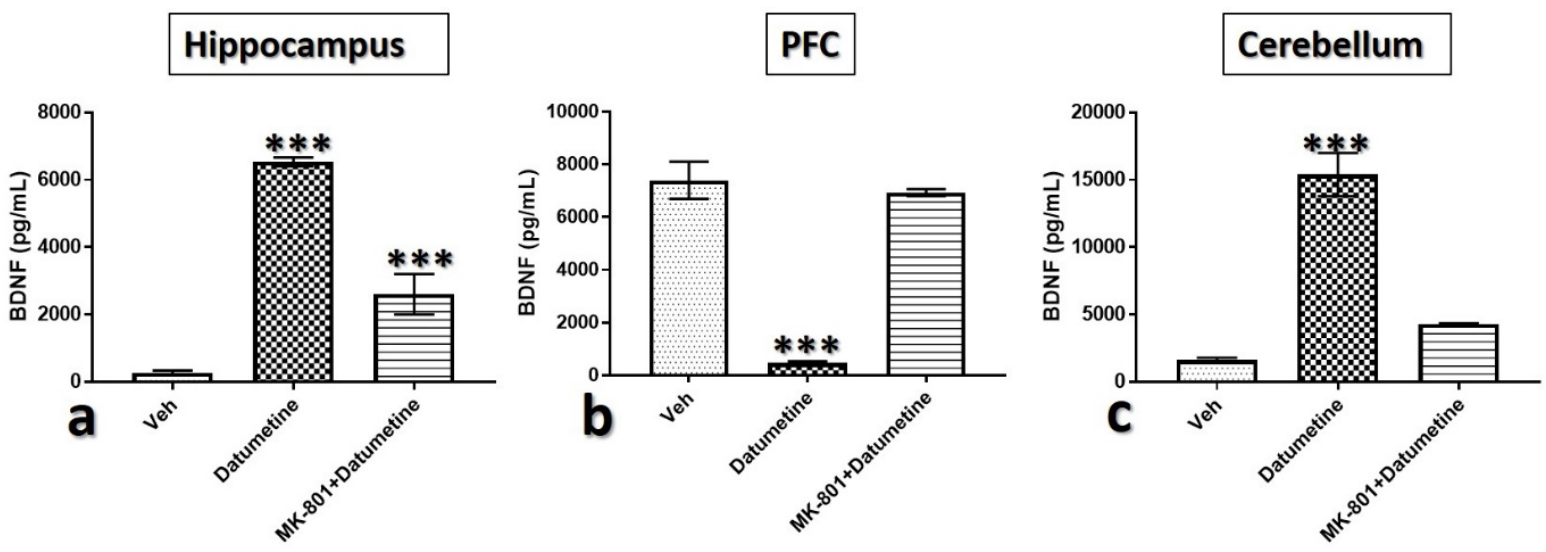

Figure 4. Graphical representation of brain-derived neurotrophic factor (BDNF) level in different brain regions

NEUR SCIENCE

A) Hippocampal level of BDNF was significantly (*** $<0.001$ ) higher in datumetine and MK-801 pre-treated mice than Veh mice, while MK-801 pre-treated mice still showed a significant reduction in BDNF than datumetine.

B) PFC BDNF level was significantly $(* *$ P $<0.001)$ reduced in datumetine mice than Veh and MK-801 pre-treated mice but no significant difference between MK-801+datumetine and Veh mice.

C) Cerebellar BDNF level was significantly higher in Datumetine mice than Veh and MK-801 pre-treated mice while no significant difference was observed between MK-801+datumetine and Veh mice.

(Figures $4 \mathrm{~A}$ and $4 \mathrm{C}$ ). Mice pre-treated with MK801 before datumetine reversed the increased BDNF level relative to datumetine although still significantly $(* * * \mathrm{P}<0.001)$ higher than Veh mice in the hippocampus (Figure $4 \mathrm{~A}$ ) but not significant in the cerebellum (Figure 4 C). Datumetine significantly $(* * * \mathrm{P}<0.001)$ decrease BDNF level in the PFC compared to Veh and MK-801 pre-treated mice (Figure $4 \mathrm{~B}$ ).

Datumetine differentially alters the glutamate system in different brain regions

The expression of neurons using anti-NeuN, glutamate terminals using anti-vGlut1 and NMDAR 2A subtype using anti-GluN2A and or NMDAR 2B subtype using anti-GluN2B were co-localized in the tissue using triple stain IHC kit gotten from Abcam USA where neurons were shown in brown, vGlut1 in red/magenta and or green, GluN2A in green and GluN2B in red.

In the hippocampus (Figure $5 \mathrm{~A}$ ), neurons were seen in the granular cell layer of the hippocampus with little expression in the molecular layer of the hippocampus. No significant difference was observed in the expression of neurons across the group, indicating that single datumetine exposure does not affect neuron distribution. vGlut1 expressions were more visible in the hippocampus granular cell layer, localized as droplets in the cytoplasm with neurons across the groups. Quantification showed a steady increase in the expression of vGlut1 positive cells in datumetine and MK-801 pre-treated mice compared to the control group but no significant difference was observed between groups. GluN2A expression was few in the granular cell layer of the hippocampus as a green line surrounding a cell (membrane) and more in the molecular layer of the hippocampus (location of dendrites) in all experimental groups, quantification showed that mice exposed to datumetine and pre-treated with MK-801 showed increase GluN2A expression compared to the control but no significant difference was observed between groups. GluN2B was observed more in the granular cell layer of the cornu ammonis (CA) and dentate gyrus (DG) of the hippocampus than in the molecular layer unlike GluN2A in the groups (Figure $6 \mathrm{~A}$ ). The expressions were mostly surrounding neurons, indicating their extra-synaptic location. Quantification showed that mice exposed to datumetine and those pre-treated with MK-801 showed a significant reduction in the expression of GluN2B compared to Veh.

In the PFC (Figure $5 \mathrm{~B}$ ), neurons are expressed in all the layers of the PFC across the experimental groups, similarly, quantification showed no significant difference in NeuN expression among the experimental groups. vGlut1 expression is also evidently expressed as tiny red/magenta droplets in the cytoplasm of the neurons in all experimental groups. Quantification reveals that datumetine and MK-801+ datumetine mice have significantly increased vGlut1 expression compared to Veh mice. While GluN2A expression is evident as green lines circling neuron (membrane) and part of the molec- 


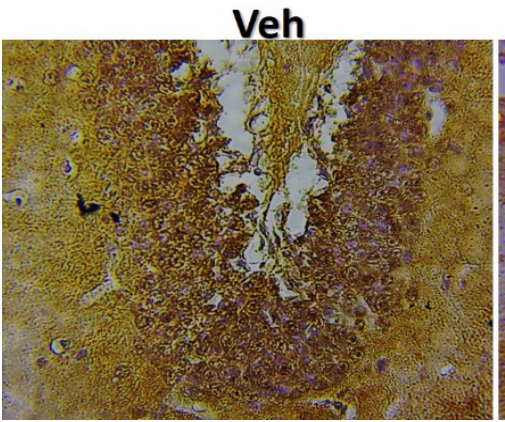

a

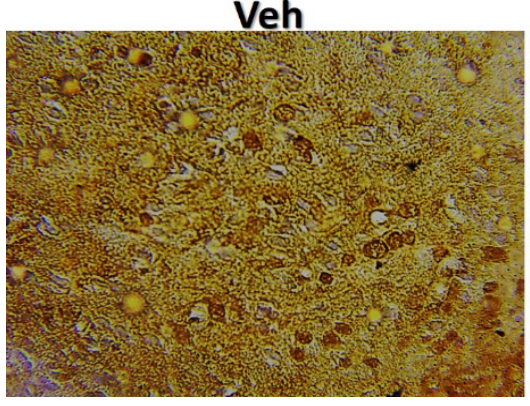

b

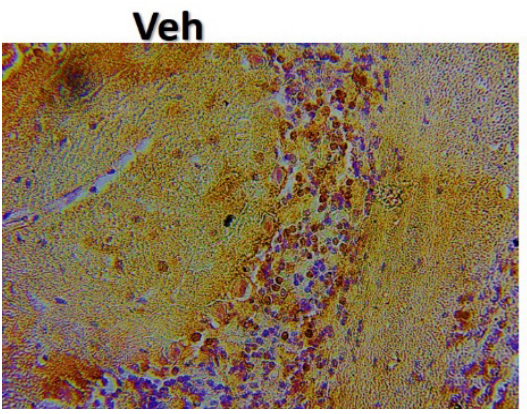

C

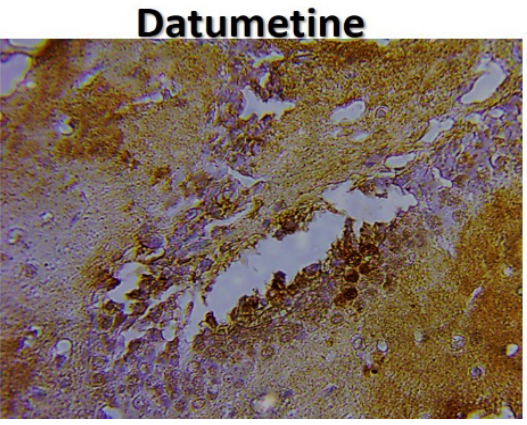

Datumetine

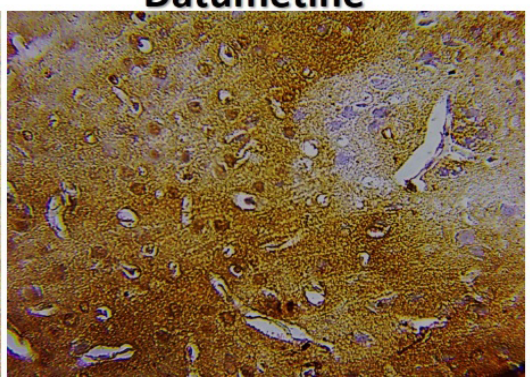

MK-801+Datumetine
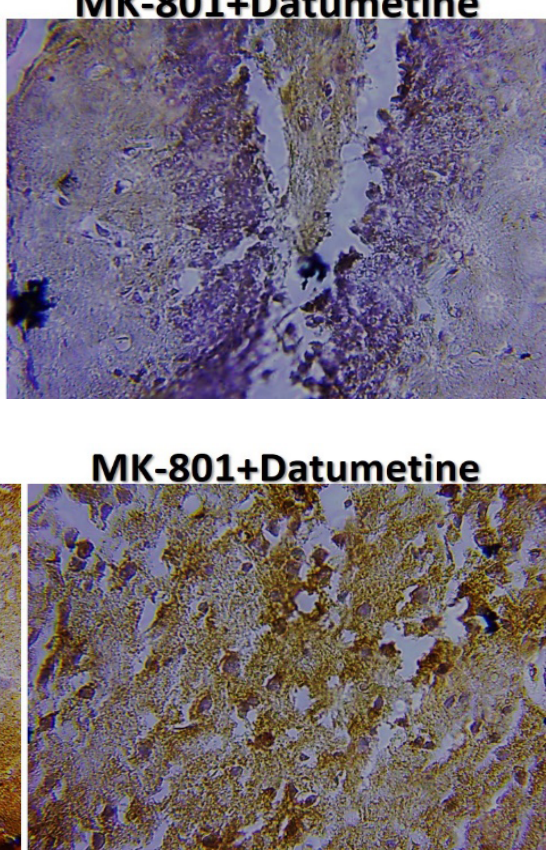

MK-801+Datumetine
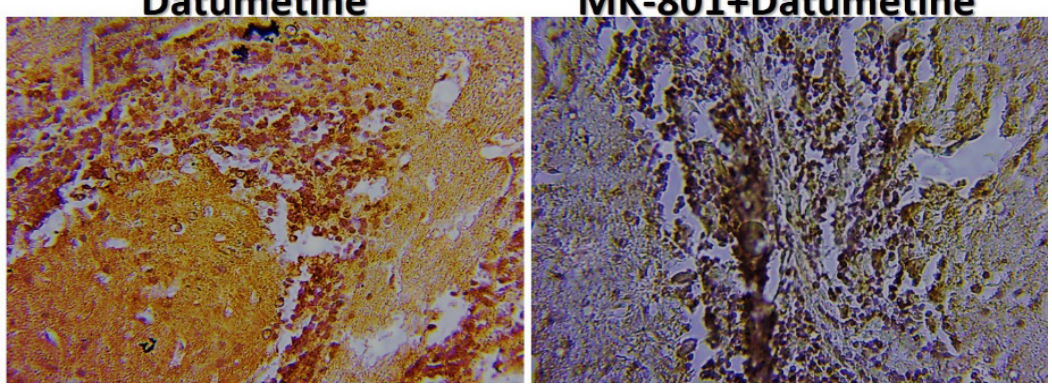

NEUR SCIENCE

Figure 5. Representative immunohistochemical slide

A) Dentating gyrus of hippocampus, B) The prefrontal cortex (PFC), and C) The cerebellar cortex of experimental animals at x40 objectives showing the expression of NeuN (brown), vGlut1 (Red) and GluN2A (green)

Datumetine treatment increases the expression of vGlut1 and GluN2A in all region of the brain observed.

ular layer in all experimental groups, also quantification showed a significant increase in expression of GluN2A in datumetine and MK-801+ datumetine mice compared to Veh mice. GluN2B expression was observed in all layers of the PFC via scanty in the molecular layer, unlike GluN2A. The expressions were seen with neurons at the membrane part of the neurons suggesting their extra-synaptic location (Figure 6 B). Also, a significant increase is observed in the expression of GluN2B in the PFC of datumetine and MK-801+datumetine mice compared to Veh mice.

In the cerebellum (Figure $5 \mathrm{C}$ ), the expression of neurons was evident in the granular cell layer and Purkinje cell layer in all experimental mice, no significant changes in the expression of NeuN among groups were observed upon quantification. vGlut1 was revealed in some cells of the granular cell layer of the cerebellum in all experimental groups, which was significantly increased in datumetine mice only compared to other groups. While GluN2A was mostly expressed in the dendrites of the Purkinje cell of the cerebellum and little expression was observed in the granular cell layer in all the experimental groups, although quantification showed no significant difference in GluN2A expression. Unlike GluN2A, which was more evident only at the dendrite of Purkinje cells, the expression of GluN2B was observed in the granular and Purkinje cell layers. The 

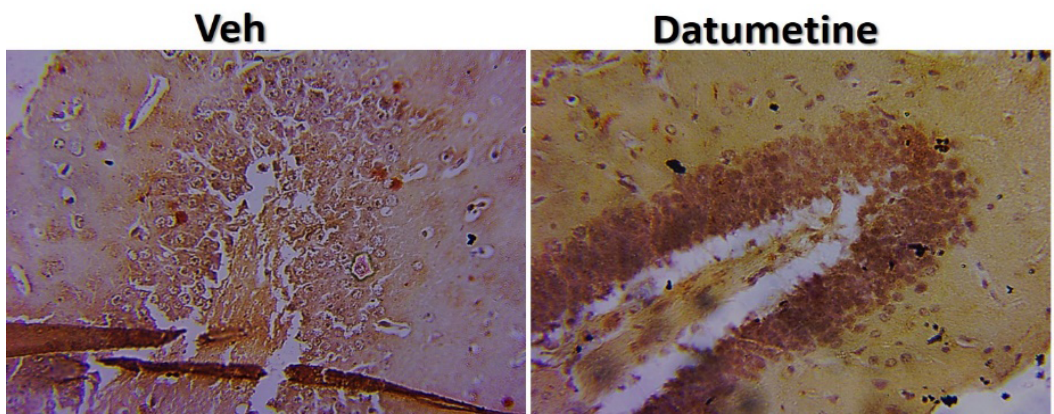

MK-801+Datumetine

a

Veh

Datumetine

MK-801+Datumetine
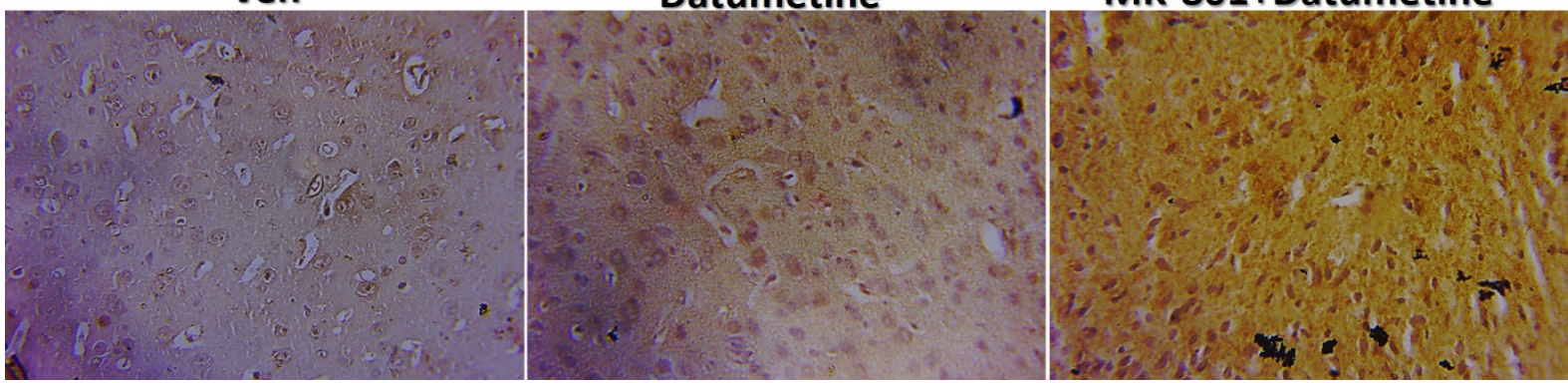

b

Veh

Datumetine
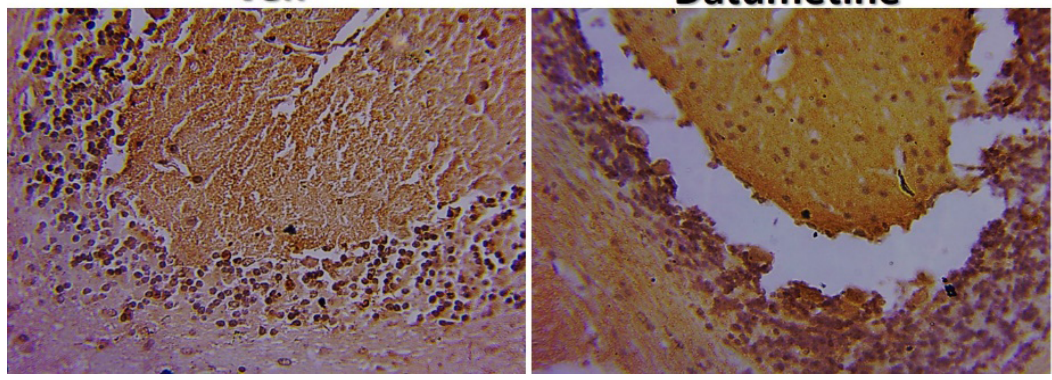

MK-801+Datumetine

C

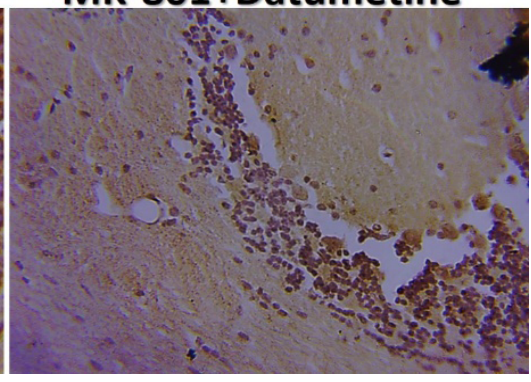

NEUR SCIENCE

Figure 6. Representative immunohistochemical slide

A) Dentating gyrus of hippocampus, B)The prefrontal cortex (PFC), and C) The cerebellar cortex of experimental animals at x40 objectives showing the expression of NeuN (brown), vGlut1 (green) and GluN2B (red)

Datumetine treatment increases GluN2B expression in the PFC and cerebellar cortex while it was reduced in hippocampus than the Veh group.

expression of GluN2B was seen around some granular cells and Purkinje cell bodies in all groups suggesting an extra-synaptic location (Figure 6 C). Quantification data showed that only mice exposed to MK-801 and datumetine showed a significant reduction in the expression of GluN2B compared to other groups.

All quantification data are supplied in the supplementary file.

\section{Discussion}

We have previously reported that datumetine, an alkaloid from Datura metel (Siddiqui et al., 1986), has an affinity to bind to NMDAR at its orthosteric and allosteric binding sites (under review). We also reported that exposure of C57BL/6 mice to datumetine for 14 days induces a seizure-like behaviour in the mice, altered NMDAR signaling, and hippocampal neurotransmitter system (Ishola et al., 2020). Thus, we hypothesize that datumetine mimics glutamate in the brain, and the observed effect is similar to glutamate excitotoxicity.

NMDAR is reported to play a diverse role in both cell survival and death (Bartlett \& Wang, 2013; Sattler et al., 2000; X. Zhou et al., 2013) there have been few attempts to correlate these important areas of research. Here we review current knowledge of the various mechanisms 
of NMDAR-dependent synaptic plasticity that are shared with neuronal survival and death, while drawing comparisons with the proneurotrophin/ neurotrophin receptor and intracellular signaling systems. Our conclusion is that NMDAR-dependent LTP and long-term depression (LTD. Thus, this study focused on the NMDAR synaptic-nuclear signaling pathway (Wang \& Peng, 2016). NMDAR activation allows for an influx of $\mathrm{Ca}^{2+}$ into the cell, which activates diverse intracellular signaling pathways (Dore et al., 2017; Hardingham \& Bading, 2003; Horak et al., 2017; Yuen et al., 2005) the interactions between them are essentially unknown. Here we show that serotonin, by activating 5-HT 1A receptors, inhibited NMDA receptormediated ionic and synaptic currents in PFC pyramidal neurons, and the NR2B subunit-containing NMDA receptor is the primary target of 5-HT1A receptors. This effect of 5-HT 1A receptors was blocked by agents that interfere with microtubule assembly, as well as by cellular knockdown of the kinesin motor protein KIF17 (kinesin superfamily member 17 . One of the associated molecules of NMDAR is CamKII $\alpha$ (Cho et al., 2012; Kennedy, 2010) a glutamate receptor, is the predominant molecular device for controlling synaptic plasticity and memory function. The NMDA receptor is specialized to initiate and control changes in synaptic strength, based on the firing patterns of the synapse. This chapter discusses the structure and function of the signaling complex assembled in the cytosol around the NMDA receptor.

The NMDA receptor is assembled from four distinct subunits that fall into structural and functional classes. The signaling complex that assembles around this tail does not appear to be a rigid structure with a fixed stoichiometry. Instead, it is believed to be assembled stochastically by associations with a set of scaffold proteins, and, in some instances, by direct association of the tails of the NMDA receptor with signaling molecules. Two features of activity-dependent plasticity at excitatory synapses in the central nervous system are central to learning mechanisms in the brain and have been the focus of many recent studies. One is the tight dependence of synaptic plasticity on I"spiketimingl" of pre- and postsynaptic neurons. The second is "metaplasticity," or the adjustment of the sensitivity and sign (LTP or LTD. Due to calcium influx by NMDAR activation, CamKII $\alpha$ gets phosphorylated at threonine 286 (Cho et al., 2012; Qi et al., 2020) hippocampal place cell stability and spatial learning. Additionally, there is evidence from hippocampal electrophysiological slice studies that this kinase has a role in regulating ion channels that control neuronal excitability. Here, we report in vivo single unit studies, with $\alpha$-CaMKII mutant mice, in which threonine 305 was replaced with an aspartate $(\alpha-\mathrm{CaMKII}$ T305D mutants. In this experiment, we observed that the
CamKII $\alpha$ level was reduced in the hippocampus and PFC of datumetine-exposed mice compared to the Veh mice. Although this difference is not significantly different compared to the mice treated with the vehicle solution. The reduction observed may be due to the phosphorylation of CamKII $\alpha$ caused by NMDAR activation from datumetine as previously reported (Shrestha et al., 2019) $\mathrm{Ca}^{++}$transient produced by glutamatergic ionotropic neurotransmission facilitates $\mathrm{Ca}^{++}$-Calmodulin-dependent kinase 2 (CaMKII that a negative correlation is observed between the level of CamKII- $\alpha$ and pCamKII-T286. Blocking NMDAR with MK-801 before datumetine treatment indicates that the effect of datumetine was reversed in the PFC as the level of CamKII $\alpha$ was higher in MK-801+ datumetine mice than datumetine mice. This shows that datumetine specifically binds to NMDAR in the PFC as MK- 801 is a known NMDAR antagonist (Amalric et al., 1994; Nygard et al., 2017; Sean et al., 2013; Singh et al., 1990; Vishnoi et al., 2015; Xue et al., 2011) MK801, prevented increases in striatal and nigral levels of NT following both single and multiple administrations of methamphetamine. Significant attenuation of the methamphetamine-induced changes in the striatal NT system were observed with MK801 doses as low as $0.01 \mathrm{mg} / \mathrm{kg}$ per dose. In contrast, administration of NMDA caused significant increases in both striatal and nigral.NT. The NMDA-induced increase in striatal NT content, like that caused by methamphetamine, was blocked by MK801. The NT system associated with the nucleus accumbens responded in a similar manner in that MK801 $(0.1 \mathrm{mg} / \mathrm{kg}$ per dose. Although in the hippocampus, mice pre-treated with MK-801 still show a steady decrease in CamKII $\alpha$ expression level compared to datumetine-treated mice. It will be expected that MK-801 should reverse datumetine effects if datumetine truly acts via NMDAR. This contradiction may be due to the bioavailability of MK-801 since datumetine was administered 30 minutes later. Glutamate transmission plays a significant role in hippocampal functions (Vásquez et al., 2014). Blocking NMDAR may increase receptor sensitivity thereby responsible for this observed effect (Kennedy, 2010; Sapkota et al., 2019) a variety of positive and negative allosteric modulators (PAMs and NAMs, respectively. A different observation was seen in the cerebellum as Datumetine animals showed an increased level of CamKII $\alpha$ while pre-treatment with MK-801 was able to reverse this effect. Cerebellar NMDAR has been reported to be unique from other brain regions of NMDAR (Galliano et al., 2018). This uniqueness may underlie the differential observation of CamKIIo level in the cerebellum. MK-801 was able to reverse this effect in the cerebellum as mice pre-treated with MK-801 showed reduced CamKII $\alpha$ compared to datumetine mice. 
$\mathrm{Ca}^{2+}$ influx into the neuron also activates cyclic adenosine monophosphate (cAMP) which then activates CREB (Montes de Oca Balderas, 2018; Nygard et al., 2017; Vásquez et al., 2014; Wang \& Peng, 2016). The level of hippocampal CREB was observed to be similar in datumetine-exposed mice with that of Veh mice, while MK-801 reversed this by increasing the CREB level. Similarly, the cerebellar level of CREB was reduced in datumetine mice which was further reduced with MK801 pre-treatment. On the contrary, CREB level was increased in the PFC of datumetine-exposed mice, while MK-801 pre-treatment reverses this effect. Activation of NMDAR often leads to phosphorylation of CREB via CamKII $\alpha$ (Wang \& Peng, 2016) or through activation of the cAMP pathway (Montes de Oca Balderas, 2018; Nygard et al., 2017; Vásquez et al., 2014). Hippocampal level of CREB in datumetine mice was similar to Veh mice, indicating that the dose of datumetine used was not neurotoxic supporting our postulations that datumetine mimics glutamate in the brain. Although the increased CREB level in the PFC of datumetine mice was reversed by MK-801, no significant difference was observed among the groups. Similarly, the cerebellar level of CREB was reduced in treated mice compared to Veh, though not significant. This disparity may be due to different NMDAR subtypes available for binding in the brain regions because different subtypes of NMDAR activate diverse signaling pathways (Cristino et al., 2015; Dong et al., 2013; Polli \& Kohlmeier, 2019; Sun et al., 2018; Xianju Zhou et al., 2013).

NMDAR activation often leads to phosphorylation of CREB at Serine 133 (Nygard et al., 2017; Wang \& Peng, 2016). We decided to evaluate the pCREB level in these different brain regions. The hippocampal pCREB level was elevated in datumetine mice compared to Veh, though not significant, while pre-treatment with MK801 did not reverse this effect. It can be postulated that Datumetine was able to activate NMDAR hereby supporting phosphorylation of CREB. MK-801 not being able to reverse this effect showed that there may be another mechanism in the brain that favours CREB phosphorylation independent of NMDAR (Rios Valentim et al., 2009; Singh et al., 1990). Another possible explanation is the bioavailability of datumetine before being metabolized since MK-801 is cleared after $2 \mathrm{~h}$ (Svalbe et al., 2019). Datumetine may remain longer than MK-801 before being metabolized thereby activating NMDAR after MK-801 metabolism. The level of pCREB was significantly elevated in the PFC of datumetine mice, supporting that datumetine was able to activate NMDAR, hereby phosphorylating CREB. This effect was reversed by MK-801, indicating that datumetine only acts in the PFC via NMDAR. Cerebellum also showed an increased level of pCREB in datumetine mice compared to Veh, but MK-801 did not reverse this effect, though no significant difference was observed among groups. This differential effect of MK-801 may be due to the uniqueness of cerebellar NMDAR kinetics and different signaling pathways initiated by NMDAR in a different region (Galliano et al., 2018; Polli \& Kohlmeier, 2019; Zhang et al., 2016).

NMDAR activation enhances synaptic plasticity through the expression of pro-survival genes (Xianju Zhou et al., 2013). One of which is BDNF. In the hippocampus, the level of BDNF was significantly high in datumetine mice than in the control, which was reversed by MK-801, although MK-801+ datumetine mice still showed a significant increase compared to Veh. This indicates that datumetine can activate NMDAR leading to synaptic plasticity, unlike our previous reports where the hippocampal BDNF level was reduced in datumetinetreated mice (under review). This pointed out that the dose of datumetine used in this study may be protective compared to the previous study. BDNF level in MK$801+$ datumetine is significantly higher than Veh in the hippocampus, which may also support the argument that datumetine remains in the brain longer than MK-801, which may account for the significant increase. A similar observation is obtained in the cerebellum as well as BDNF was significantly increased in Datumetine mice compared to Veh and MK-801+datumetine mice. On the contrary, the level of BDNF was significantly lower in the PFC of datumetine mice compared to Veh and MK801 + datumetine mice. This may be because NMDAR activation in the PFC is not coupled with BDNF expression. Another possible mechanism is that datumetine may preferentially bind to GluN2B in the PFC and this subtype of NMDAR is usually associated with long-term depression (LTD) (Dong et al., 2013; Sun et al., 2018; Zhou et al., 2013; Zhu et al., 2016). The cerebellum also showed a similar trend with the hippocampus as mice exposed to datumetine showed a significant elevation of BDNF compared to Veh and MK-801+ datumetine mice.

Immunohistochemistry results showed no observable changes in neuron distribution in all the brain regions observed in the experimental groups. This indicates that the dose used in this study is not neurotoxic, contrary to the previous report that datumetine induces hippocampal neuronal loss with activation of astrocytes and microglia (Ishola et al., 2020). Expression of vGlut1 was increased in all brain regions indicating that datumetine enhances glutamate transmission even at this low dose, 
similar to our earlier report (Ishola et al., 2020). Two major NMDAR subtypes found to be widely studied were evaluated. GluN2A expression was increased in all treated groups compared to the Veh mice, while GluN2B expression was reduced in all treated groups compared to the Veh mice. It may be postulated that this differential expression points that datumetine preferentially bind to the GluN2B subtype of NMDAR than the GluN2A subtype; however, further research is still needed to determine whether datumetine preferentially binds to different subtypes of NMDAR in the brain.

\section{Conclusion}

We concluded that a low dose of datumetine activates NMDAR thereby enhancing its intracellular signalling in different brain regions. This may serve as a therapeutic potential for enhancing NMDAR activity in the brain.

\section{Ethical Considerations}

\section{Compliance with ethical guidelines}

This work was approved by the Ethical Review Committee of the University of Ilorin (Code UERC/ $\mathrm{BMS} / 108)$.

\section{Funding}

This study was collated from the undergraduate dissertation of Isaac Chukwunwike Abanum, Adesola Akorede Adeyemi, Caleb Kenechukwu Faleye, Jane Babale Martins, Nnenna Chimdalu Ogbe, Toluwalase Cherish Ogundipe, Karen Ezichi Okewulonu, Umo Emmanuel Okon, and Deborah Irenoise Ovbude submitted to the Department of Anatomy, Faculty of Basic Medical Sciences, College of Medicine and Health Sciences, Afe Babalola University.

\section{Authors' contributions}

Conceptualisation: Azeez Olakunle Ishola, Adedeji Enitan Adetunj, Moyosore Salihu Ajao; Acquire and analyse data: Azeez Olakunle Ishola, Adedeji Enitan Adetunj, Richard Yomi Akele, Naeemat Tolulope Omotade, Isaac Chukwunwike Abanum, Adesola Akorede Adeyemi, Caleb Kenechukwu Faleye, Jane Babale Martins, Nnenna Chimdalu Ogbe, Toluwalase Cherish Ogundipe, Karen Ezichi Okewulonu, Umo Emmanuel Okon, and Deborah Irenoise Ovbude; Manuscript prepartation: Azeez Olakunle Ishola, Adedeji Enitan Adetunj, Richard Yomi Akele and Moyosore Salihu Ajao.

\section{Conflict of interest}

The authors declared no conflict of interest.

\section{Acknowledgments}

The authors appreciate O. M. Ogundele of LSU-SVM USA for the datumetine gift.

\section{References}

Amalric, M., Ouagazzal, A., Baunez, C., \& Nieoullon, A. (1994). Functional interactions between glutamate and dopamine in the rat striatum. Neurochemistry International, 25(2), 123-131. [DOI:10.1016/0197-0186(94)90031-0] [PMID]

Bartlett, T.E., \& Wang, Y. T. (2013). The intersections of NMDARdependent synaptic plasticity and cell survival. Neuropharmacology, 74, 59-68. [DOI:10.1016/j.neuropharm.2013.01.012] [PMID]

Cho, J., Bhatt, R., Elgersma, Y., \& Silva, A. J. (2012). a-Calcium calmodulin kinase II modulates the temporal structure of hippocampal bursting patterns. PloS One, 7(2), e31649. [DOI:10.1371/journal.pone.0031649] [PMID] [PMCID]

Cristino, L., Luongo, L., Squillace, M., Paolone, G., Mango, D., \& Piccinin, S., et al. (2015). D-Aspartate oxidase influences glutamatergic system homeostasis in mammalian brain. Neurobiology of Aging, 36(5), 1890-1902. [DOI:10.1016/j.neurobiolaging.2015.02.003] [PMID]

Das, D., Biswal, S., Barhwal, K. K., Chaurasia, O. P., \& Hota, S, K. (2018). Kaempferol inhibits Extra-synaptic NMDAR-Mediated Downregulation of TRk $\beta$ in rat hippocampus during hypoxia. Neuroscience, 392, 77-91. [DOI:10.1016/j.neuroscience.2018.09.018] [PMID]

Dong, Z., Bai, Y., Wu, X., Li, H., Gong, B., \& Howland, J. G., et al. (2013). Hippocampal long-term depression mediates spatial reversal learning in the Morris water maze. Neurophar macology, 64, 65-73. [DOI:10.1016/j.neuropharm.2012.06.027] [PMID]

Dore, K., Stein, I. S., Brock, J. A., Castillo, P. E., Zito, K., \& Sjöström, P. J. (2017). Unconventional NMDA receptor signaling.The Journal of Neuroscience, 37(45), 10800-10807. [DOI:10.1523/JNEUROSCI.1825-17.2017] [PMID] [PMCID]

Galliano, E., Schonewille, M., Peter, S., Rutteman, M., Houtman, S., \& Jaarsma, D., et al. (2018). Impact of NMDA receptor overexpression on cerebellar purkinje cell activity and motor learning. ENeuro, 5(1), ENEURO.0270-17.2018. [DOI:10.1523/ ENEURO.0270-17.2018] [PMID] [PMCID]

Hardingham, G. E., \& Bading, H. (2003). The Yin and Yang of NMDA receptor signalling. Trends in Neurosciences, 26(2), 8189. [DOI:10.1016/S0166-2236(02)00040-1] [PMID] 
Henson, M. A., Tucker, C. J., Zhao, M., \& Dudek, S. M. (2017). Long-term depression-associated signaling is required for an in vitro model of NMDA receptor-dependent synapse pruning. Neurobiology of Learning and Memory, 138, 39-53. [DOI:10.1016/j.nlm.2016.10.013] [PMID] [PMCID]

Horak, M., Holubova, K., Nepovimova, E., Krusek, J., Kaniakova, M., \& Korabecny, J., et al. (2017). The pharmacology of tacrine at N-methyl-D-aspartate receptors. Progress in Neuro-Psychopharmacology and Biological Psychiatry, 75, 54-62. [DOI:10.1016/j.pnpbp.2017.01.003] [PMID]

Ishola, A. O., Imam, A., \& Ajao, M. S. (2020). Datumetine exposure alters hippocampal neurotransmitters system in C57BL/6 mice. Drug and Chemical Toxicology, 45(2), 785-798. [DOI:10.1080/01480545.2020.1776315] [PMID]

Ishola, A. O., Imam, A., \& Ajao, M. S. (2021). Effects of datumetine on hippocampal NMDAR activity. Toxicology Reports, 8, 11311142. [DOI:10.1016/j.toxrep.2021.05.009] [PMID] [PMCID]

Kennedy, M. B. (2010). Synaptic NMDA-receptor signaling complex. In R.A. Bradshaw, \& E. A. Dennis (Eds.), Handbook of cell signaling. Cambridge: Academic Press. [DOI:10.1016/B978-012-374145-5.00158-3]

Lüscher, C., \& Malenka, R. C. (2012). NMDA receptor-dependent long-term potentiation and long-term depression (LTP/ LTD). Cold Spring Harbor Perspectives in Biology, 4(6), a005710. [Link]

Montes de Oca Balderas, P. (2018). Flux-independent NMDAR signaling: Molecular mediators, cellular functions, and complexities. International Journal of Molecular Sciences, 19(12), 3800. [DOI:10.3390/ijms19123800] [PMID] [PMCID]

Moritz, D. J., Fox, P. J., Luscombe, F. A., \& Kraemer, H. C. (1997). Neurological and psychiatric predictors of mortality in patients with Alzheimer disease in California. Archives of Neurology, 54(7), 878-885. [DOI:10.1001/archneur.1997.00550190066016] [PMID]

Ni, M., Zhang, J., Huang, L., Liu, G., \& Li, Q. (2018). A Rhokinase inhibitor reverses learning and memory deficits in a Rat model of chronic cerebral ischemia by altering Bcl-2/ Bax-NMDAR signaling in the cerebral cortex. Journal of Pharmacological Sciences, 138(2), 107-115. [DOI:10.1016/j. jphs.2018.08.012] [PMID]

Nygard, S. K., Klambatsen, A., Balouch, B., Quinones-Jenab, V., \& Jenab, S. (2017). NMDAR dependent intracellular responses associated with cocaine conditioned place preference behavior. Behavioural Brain Research, 317, 218-225. [DOI:10.1016/j. bbr.2016.09.047] [PMID] [PMCID]

Polli, F. S., \& Kohlmeier, K. A. (2019). Alterations in NMDARmediated signaling within the laterodorsal tegmental nucleus are associated with prenatal nicotine exposure. Neuropharmacology, 158, 107744. [DOI:10.1016/j.neuropharm.2019.107744] [PMID]

Qi, F., Liu, T., Zhang, X., Gao, X., Li, Z., \& Chen, L., et al. (2020). Ketamine reduces remifentanil-induced postoperative hyperalgesia mediated by CaMKII-NMDAR in the primary somatosensory cerebral cortex region in mice. Neuropharmacology, 162, 107783. [DOI:10.1016/j.neuropharm.2019.107783] [PMID]
Reiner, A., \& Levitz, J. (2018). Glutamatergic signaling in the central nervous system: Ionotropic and metabotropic receptors in concert. Neuron, 98(6), 1080-1098. [PMID] [PMCID]

Rios Valentim, S. J., Jr, Gontijo, A. V., Peres, M. D., Rodrigues, L. C., \& Nakamura-Palacios, E. M. (2009). D1 dopamine and NMDA receptors interactions in the medial prefrontal cortex: Modulation of spatial working memory in rats. Behavioural Brain Research, 204(1), 124-128. [PMID]

Sapkota, K., Dore, K., Tang, K., Irvine, M., Fang, G., \& Burnell, E. S., et al. (2019). The NMDA receptor intracellular C-terminal domains reciprocally interact with allosteric modulators. Biochemical Pharmacology, 159, 140-153. [DOI:10.1016/j. bcp.2018.11.018] [PMID] [PMCID]

Sattler, R., Xiong, Z., Lu, W. Y., MacDonald, J. F., \& Tymianski, M. (2000). Distinct roles of synaptic and extrasynaptic NMDA receptors in excitotoxicity. The Journal of Neuroscience , 20(1), 22-33. [DOI:10.1523/jneurosci.20-01-00022.2000] [PMID] [PMCID]

McKay, S., Bengtson, C. P., Bading, H., Wyllie, D. J., \& Hardingham, G. E. (2013). Recovery of NMDA receptor currents from MK-801 blockade is accelerated by $\mathrm{Mg} 2+$ and memantine under conditions of agonist exposure. Neuropharmacology, 74, 119-125. [DOI:10.1016/j.neuropharm.2013.01.024] [PMID] [PMCID]

Shrestha, A., Sultana, R., Lee, C. C., \& Ogundele, O. M (2019). SK Channel modulates synaptic plasticity by tuning CaMKIIa/ $\beta$ Dynamics. Frontiers in Synaptic Neuroscience, 11, 18. [DOI:10.3389/fnsyn.2019.00018] [PMID] [PMCID]

Siddiqui, S., Sultana, N., Ahmed, S. S., \& Haider, S. I. (1986). Isolation and structure of a new alkaloid datumetine from the leaves of datura metel. Journal of Natural Products, 49(3), 511513. [DOI:10.1021/np50045a023]

Singh, N. A., Bush, L. G., Gibb, J. W., \& Hanson, G. R. (1990) Dopamine-mediated changes in central nervous system neurotensin systems: A role for NMDA receptors. European Journal of Pharmacology, 187(3), 337-344. [DOI:10.1016/00142999(90)90361-9] [PMID]

Sun, Y., Xu, Y., Cheng, X., Chen, X., Xie, Y., \& Zhang, L., et al. (2018). The differences between GluN2A and GluN2B signaling in the brain. Journal of Neuroscience Research, 96(8), 14301443. [DOI:10.1002/jnr.24251] [PMID]

Svalbe, B., Grinberga, S., Stelfa, G., Vavers, E., Zvejniece, B., \& Sevostjanovs, E., et al. (2019). Data on analysis of MK-801 bioavailability in mouse plasma and brain tissue by ultra-performance liquid chromatography-tandem mass spectrometry. Data in Brief, 27, 104623. [PMID] [PMCID]

Vásquez, C. E., Riener, R., Reynolds, E., \& Britton, G. B. (2014). NMDA receptor dysregulation in chronic state: A possible mechanism underlying depression with BDNF downregulation. Neurochemistry International, 79, 88-97. [DOI:10.1016/j. neuint.2014.09.007] [PMID]

Vishnoi, S., Raisuddin, S., \& Parvez, S. (2015). Modulatory effects of an NMDAR partial agonist in MK-801-induced memory impairment. Neuroscience, 311, 22-33. [DOI:10.1016/j.neuroscience.2015.10.008] [PMID] 
Wang, H., \& Peng, R. Y. (2016). Basic roles of key molecules connected with NMDAR signaling pathway on regulating learning and memory and synaptic plasticity. Military Medical Research, 3(1), 26. [DOI:10.1186/s40779-016-0095-0] [PMID] [PMCID]

Xue, J. G., Masuoka, T., Gong, X. D., Chen, K. S., Yanagawa, Y., \& Law, S. K., et al. (2011). NMDA receptor activation enhances inhibitory GABAergic transmission onto hippocampal pyramidal neurons via presynaptic and postsynaptic mechanisms. Journal of Neurophysiology, 105(6), 2897-2906. [DOI:10.1152/jn.00287.2010] [PMID]

Yuen, E. Y., Jiang, Q., Chen, P., Gu, Z., Feng, J., \& Yan, Z. (2005). Serotonin 5-HT1A receptors regulate NMDA receptor channels through a microtubule-dependent mechanism. The Journal of Neuroscience, 25(23), 5488-5501. [DOI:10.1523/JNEUROSCI.1187-05.2005] [PMID] [PMCID]

Zhang, Q., Liu, Q., Li, T., Liu, Y., Wang, L., \& Zhang, Z., et al. (2016). Expression and colocalization of NMDA receptor and FosB/ $\delta$ FosB in sensitive brain regions in rats after chronic morphine exposure. Neuroscience Letters, 614, 70-76. [DOI:10.1016/j.neulet.2015.11.052] [PMID]

Zhou, X., Hollern, D., Liao, J., Andrechek, E., \& Wang, H. (2013). NMDA receptor-mediated excitotoxicity depends on the coactivation of synaptic and extrasynaptic receptors. Cell Death and Disease, 4(3), e560. [PMID] [PMCID]

Zhou, X., Ding, Q., Chen, Z., Yun, H., \& Wang, H. (2013). Involvement of the GluN2A and GluN2B subunits in synaptic and extrasynaptic N-methyl-D-aspartate receptor function and neuronal excitotoxicity. The Journal of Biological Chemistry, 288(33), 24151-24159. [PMID] [PMCID]

Zhu, G., Liu, Y., Wang, Y., Bi, X., \& Baudry, M. (2015). Different patterns of electrical activity lead to Long-Term potentiation by activating different intracellular pathways. The Journal of Neuroscience: The Official Journal of the Society for Neuroscience, 35(2), 621-633. [PMID] [PMCID]

Zhu, S., Stein, R. A., Yoshioka, C., Lee, C. H., Goehring, A., \& Mchaourab, H. S., et al. (2016). Mechanism of NMDA Receptor Inhibition and Activation. Cell, 165(3), 704-714. [PMID] [PMCID] 
This Page Intentionally Left Blank 